\title{
Remarks on Feedforward Circuits, Adaptation, and Pulse Memory
}

\author{
Eduardo D. Sontag \\ Department of Mathematics \\ Rutgers University \\ New Brunswick, NJ 08903
}

\begin{abstract}
This note studies feedforward circuits as models for perfect adaptation to step signals in biological systems. A global convergence theorem is proved in a general framework, which includes examples from the literature as particular cases. A notable aspect of these circuits is that they do not adapt to pulse signals, because they display a memory phenomenon. Estimates are given of the magnitude of this effect.
\end{abstract}

\section{Introduction}

Feedforward circuits have been often proposed for adaptation to constant signals in biological systems. Indeed, the review paper [26] gives a chemical reaction model, called there a "sniffer" and shown in Figure 1, as the paradigm for perfect adaptation. The chemical species $S$ acts as a "sig-

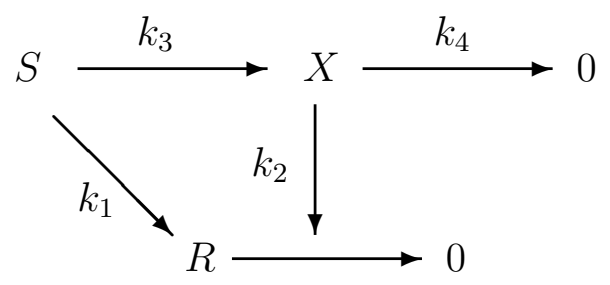

Figure 1: "Sniffer" network, from [26]

nal", and the species $R$ is viewed as a "response" element. The third species, $X$, is an intermediate species. The species $S$ directly helps promote the formation of $R$ (arrow labeled " $k_{1}$ "), and also the formation of $X$ (arrow labeled " $k_{3}$ "). On the other hand, $X$ also enhances degradation of the species $R$ (vertical arrow labeled " $k_{2}$ "), and thus $S$ also acts through $X$ as an inhibitor of $R$, counteracting the positive direct effect. This "incoherent" counterbalance between a positive and a negative effect gives rise to a regulation property.

Mathematically, the model is described in [26] as a system of two coupled differential equations 
for the concentrations of the substances in question, using mass-action kinetics:

$$
\begin{aligned}
\dot{x} & =k_{3} s-k_{4} x \\
\dot{r} & =k_{1} s-k_{2} x r
\end{aligned}
$$

where dot is used to indicate time derivative. The key fact is that in steady state and for nonzero constant signals $S$, the concentration of $R$ equals $\frac{k_{1} k_{4}}{k_{2} k_{3}}$, and this value is independent of the actual value of $S$. (This follows simply by setting the right-hand sides of the equations to zero and solving for $r$.)

Similar constructions have been given in other biological investigations of adaptation, notably in [30], where various possible chemical networks are proposed for modeling adaptation by the chemotaxis pathway of Dictyostelium.

A common feature of these models is that they have the form of a stable linear system which in turn drives a one-dimensional (generally nonlinear) system, whose state-variable represents the response that should adapt. The interconnection is set up so that the whole system becomes a "feedforward circuit" [1].

\section{General framework}

The strength of the external input signal (a non-negative real number) will be denoted by $u$, the state of the linear system (an $n$-dimensional vector) by $x$, and the state of the driven response (a real number) by $y$. Then, a mathematical description of the evolution of concentrations of the various signals is given by a system of $n+1$ differential equations as follows:

$$
\begin{aligned}
& \dot{x}=A x+b u \\
& \dot{y}=c(y) x+d(y) u
\end{aligned}
$$

where $A$ and $b$ are a constant stable matrix and column vector respectively, $A \in \mathbb{R}^{n \times n}, b \in \mathbb{R}^{n \times 1}$, and $c(y)$ and $d(y)$ are continuous functions of $y$ so that, for each $y, c(y) \in \mathbb{R}^{1 \times n}$ and $d(y) \in \mathbb{R}$. System (2] is a system with inputs and outputs, in the standard sense of control theory [23]. The state variables $x(t)$ and $y(t)$ take values in some subsets $\mathcal{X} \subseteq \mathbb{R}^{n}$ and $\mathcal{Y} \subseteq \mathbb{R}$ respectively, where $\mathcal{Y}$ is a closed, possibly unbounded, interval. The sets $\mathcal{X}$ and $\mathcal{Y}$ can be used in order to impose nonnegativity and/or mass conservation constraints. Enough regularity is assumed so that, for every non-negative constant input $u_{0}$, and every initial condition $\left(x_{0}, y_{0}\right) \in \mathcal{X} \times \mathcal{Y}$, the equations (2) have a unique solution $(x(t), y(t)) \in \mathcal{X} \times \mathcal{Y}$ defined for all $t \geq 0$.

For example, in the "sniffer" reactions (1) from [26], and writing $u, x$, and $y$ instead of $s, x$, and $r$ respectively, one has $\mathcal{X}=\mathcal{Y}=\mathbb{R}_{\geq 0}, A=-k_{4}, b=k_{3}, c(y)=-k_{2} y$, and $d(y)=k_{1}$ (constant). In these notations Equations (1) become:

$$
\begin{aligned}
& \dot{x}=-k_{4} x+k_{3} u \\
& \dot{y}=-k_{2} x y+k_{1} u .
\end{aligned}
$$

\section{Adaptation to step inputs}

For each nonzero constant input $u_{0}$, the steady states $\left(x_{0}, y_{0}\right)$ of the system (2) are obtained by first setting $\dot{x}=0$, which gives $x_{0}=-A^{-1} b u_{0}$ (the inverse is well-defined because $A$ was assumed to be 
stable, and in particular all its eigenvalues are nonzero), and then substituting into the left-hand side of the $y$-equation. There obtains the following algebraic equation:

$$
d(y)-c(y) A^{-1} b=0
$$

(after canceling out $u_{0} \neq 0$ ). A key hypothesis made from now on (and which is satisfied in all the cited examples) is that there is a unique solution $y=y_{0}$ of the algebraic equation (4), and that this solution is an asymptotically stable state for the reduced system

$$
\dot{y}=\left(d(y)-c(y) A^{-1} b\right) u_{0}
$$

that would result if $x(t)$ were already at its steady state $-A^{-1} b u_{0}$. To be precise, the following hypothesis will be imposed:

$$
\left(\exists y_{0} \in \mathcal{Y}\right)(\forall y \in \mathcal{Y})\left[\left(y-y_{0}\right)\left(d(y)-c(y) A^{-1} b\right)<0\right]
$$

(that is, $\dot{y}=\left(d(y)-c(y) A^{-1} b\right) u_{0}$ is positive when $y<y_{0}$ and negative when $\left.y>y_{0}\right)$. It is fundamental to observe that $y_{0}$ (though not, of course, $x_{0}$ ) is independent of the particular numerical value of $u_{0}$.

Proposition 2.1 shows that, assuming boundedness of trajectories, systems (2) "adapt" to nonzero constant signals $u_{0}$ ("step signals"), in the sense that all the solutions of the system (2) converge to the above steady state $\left(x_{0}, y_{0}\right)$, where $y_{0}$ is independent of $u_{0}$.

Take again as an example the equations (3) from [26], now with all $k_{i}=1$, Figure 1(b) plots the response to the piecewise constant input with nonzero values that is shown in Figure 1 (a). It is clear that this response adapts to the value $y_{0}=1$.

\section{Memory of pulse inputs}

One of the main objectives of this note is to bring attention to the following additional facts. When $u_{0}=0$, that is, in the absence of an external signal, steady states are no longer unique. Indeed, any vector of the form $(0, y)$ is a steady state. This has an important consequence for the behavior of system (2) when a pulse input is used. A pulse is defined here as an input $u$ which has the following form: $u(t)=u_{0} \neq 0$ for some interval $t \in[0, T]$, and $u(t)=0$ for $t>T$. Suppose that the interval is long enough $(T \gg 1)$ so that one may assume that $x(T)$ and $y(T)$ are (approximately) in steady state: $x(T) \approx-A^{-1} b u_{0}$ and $y(T) \approx y_{0}$. Upon removal of the external excitation at time $T$, the equations for the system become $\dot{x}=A x$ and $\dot{y}=c(y) x$ for $t>T$, with initial conditions $x(T)$ and $y(T)$, so $x(t) \approx-e^{(t-T) A} A^{-1} b u_{0}$ for $t \geq T$. The solution of $\dot{y}=c(y) x$ starts at $y_{0}$ but adds a quantity which integrates the effect of the nonzero function $x(t)$. The response may then settle to a new value which is different than the adapted value $y_{0}$.

Thus, feedforward systems for adaptation as those discussed here exhibit a "memory" effect with respect to (ideal) pulses. This phenomenon was apparently not remarked upon earlier. The present note discusses the effect and provides estimates.

To illustrate the phenomenon with the simplest possible (if not biologically meaningful) example, take $n=1, A=-1, b=1, c(y)=-1$, and $d(y)=1$. One has that $\dot{y} \approx-e^{T-t} u_{0}$, so $y(t) \rightarrow y_{0}-u_{0}<$ $y_{0}$ as $t \rightarrow \infty$ (assuming that $u_{0}>0$ ). In fact, the larger the $u_{0}$, the smaller the new asymptotic value $y_{0}-u_{0}$ is. For the nonlinear equations (3) from [26], the same phenomenon holds. Taking all

$k_{i}=1$, Figure 1(d) plots the response to a pulse of unit amplitude and Figure 1(f) plots the response 
(a)

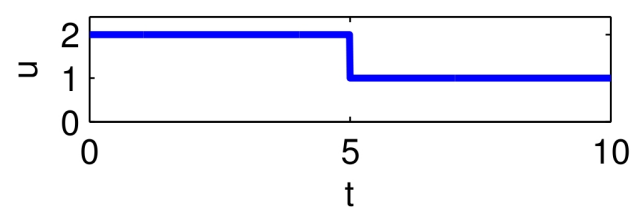

(c)

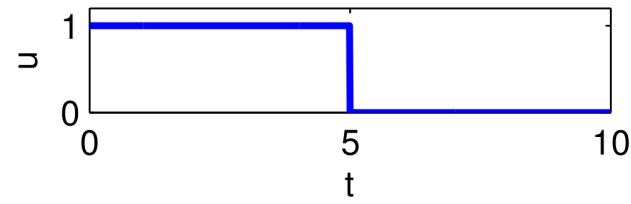

(e)

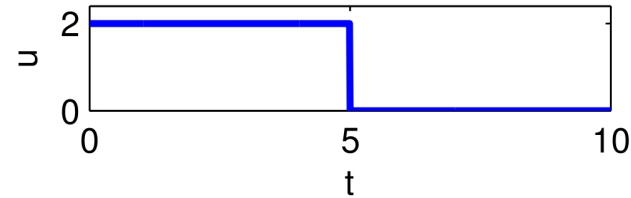

(g)

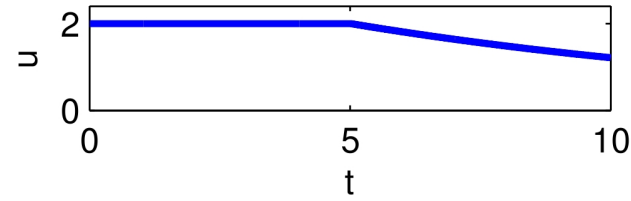

(b)

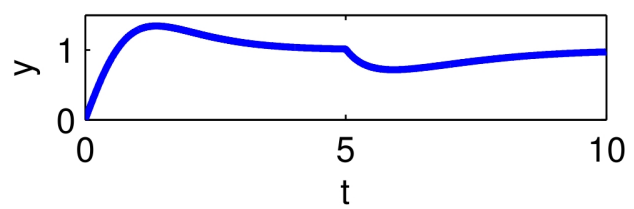

(d)

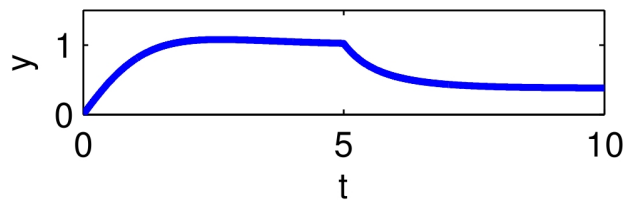

(f)

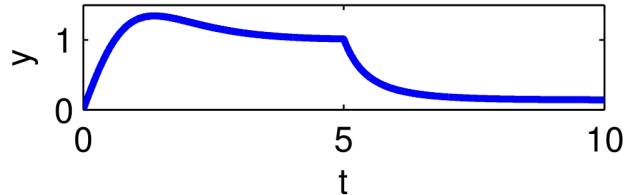

(h)

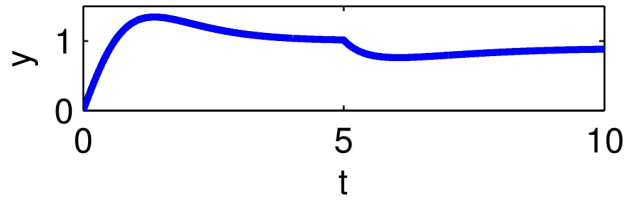

Figure 2: Plots for example discussed in text. In left column are inputs $u(t)$, in right column are responses $y(t)$. Initial conditions are $x(0)=y(0)=0 . u(t)$ is constant up to time $t=5$, switches to another constant value at time $t=5$. (a,b) $u$ switches to a nonzero value, $y$ adapts again. (c,d) Pulse of magnitude 1 : asymptotic value of $y$ is $\approx 1 / e \approx 0.37$ after pulse. (e,f) Pulse of larger magnitude 2 : asymptotic value of $y$ is smaller: $\approx 1 / e^{2} \approx 0.14$ after pulse. $(\mathrm{g}, \mathrm{h})$ Exponential decaying $u(t)=2 e^{(t-5) / 10}$ after time $t=5$ results in return to close to adapted value $y(\infty) \approx 0.9$ $(u(t)$ plotted only on interval $[0,10]$ for ease of comparison).

to a pulse of twice the amplitude. Once again, the response settles to a value that is smaller when the amplitude of the pulse was larger. The sharp cut-off of an ideal pulse plays an important role on this "memory" effect: when the input instead returns slowly enough to its baseline value, an almost-adapted response is recovered, as shown in Figure 1/(h).

\section{Feedforward motifs in systems biology}

Feedforward circuits are ubiquitous in biology, as emphasized in [1, where (incoherent) feedforward circuits were shown to be over-represented in E.coli gene transcription networks compared to other "motifs" involving three nodes. Similar conclusions apply to certain control mechanisms in mammalian cells [13. A large number of papers have been devoted to the signal-processing capabilities of the feedforward motif, notably [15] which looked into its properties as a "change detector" (essentially, sensitivity to changes in the magnitude of the input signal), and [5] which studies its optimality with respect to periodic inputs. Comparisons with other "three node" architectures with respect to the trade-off of sensitivity versus noise filtering are given in [8]. Other references on feedforward circuits include [22] (showing their over-representation at the interface of genetic and metabolic networks), [28] (classification of different subtypes of such circuits), and [10] (clas- 
sification into "time-dependent" versus "dose-dependent" biphasic responses, which are in a sense the opposite of adaptated responses). The latter reference provides a large number of additional incoherent feedforward input-to-response circuits, including: EGF to ERK activation [21, 18], glucose to insulin release [17, 19], ATP to intracellular calcium release [14, 16], nitric oxide to NF- $\kappa \mathrm{B}$ activation [20], microRNA regulation [25], and many others. Dealing specifically with adaptation properties of feedforward circuits, and in addition to the papers [26, 30], are the paper [29] on microRNA-mediated loops, and [11, which deals with the role of feedforward structures in the robust behavior in E.coli carbohydrate uptake via the carbohydrate phosphotransferase system (an analogous metabolic mechanism is also discussed in [27]).

\section{Outline of paper}

Section 2 has statements of the convergence results. Section 3 has a brief discussion of "approximate" adaptation by feedforward circuits. Section 4 shows estimates of the magnitude of the pulse memory effect. Section 5 has the proofs of the convergence results. Section 6 revisits the motivating examples and also briefly discusses the systems in [11, 29]. Finally, it is known that, under appropriate technical assumptions, perfect adaptation implies that the system may be written, after a suitable nonlinear change of coordinates, as a system in which the integral of the regulated quantify is fed-back, see for instance [6, 7, 31, 24,. This fact is not incompatible with the system being a feedforward system, as remarked in Section 7. Section 8 summarizes the results and speculates on their significance.

\section{Statements of convergence results}

The main convergence result is as follows. Note that, since the $x$-coordinate of a solution $(x(t), y(t))$ for constant $u$ always converges (because of the stability assumption on the linear system) and hence is bounded, asking that $(x(t), y(t))$ is bounded is the same as asking that $y(t)$ is.

Proposition 2.1 Suppose that Property $(\mathbf{H})$ holds. Then, for each step input $u \equiv u_{0} \neq 0$, and every initial condition, if the corresponding solution $(x(t), y(t))$ of $(2)$ is bounded, then it converges to $\left(x_{0}, y_{0}\right)$.

Boundedness is automatically satisfied if $\mathcal{Y}$ is itself a bounded interval, as is the case if mass conservation laws constrain the system dynamics. More generally, the following condition can be helpful. It strengthens Property Property $(\mathbf{H})$ for small and for large values of $y$ :

$$
\begin{aligned}
& \left(\forall u_{0}>0\right)(\exists \bar{\varepsilon}>0) \\
& \quad\left(\exists y_{2} \in \mathcal{Y}\right)\left(\forall y_{2}<y \in \mathcal{Y}\right)\left[|c(y)| \bar{\varepsilon}+u_{0}\left(d(y)-c(y) A^{-1} b\right)<0\right] \\
& \quad\left(\exists y_{1} \in \mathcal{Y}\right)\left(\forall y_{1}>y \in \mathcal{Y}\right)\left[-|c(y)| \bar{\varepsilon}+u_{0}\left(d(y)-c(y) A^{-1} b\right)>0\right]
\end{aligned}
$$

(where $|c|$ denotes the norm of the vector $c$ ) and it says that the inequality in $(\mathbf{H})$ is preserved under small enough perturbations proportional to $c(y)$, as long as $y$ is large or small enough.

Lemma 2.2 Suppose that $(\mathbf{H})$ and $\left(\mathbf{H}^{*}\right)$ are satisfied. Then, for each step input $u \equiv u_{0} \neq 0$, and every initial condition, the corresponding solution $(x(t), y(t))$ of (2) is bounded. 
From Proposition 2.1 and Lemma 2.2, there is the following immediate consequence:

Corollary 2.3 Suppose that $(\mathbf{H})$ and $\left(\mathbf{H}^{*}\right)$ are satisfied. Then, for each step input $u \equiv u_{0} \neq 0$, and every initial condition, the corresponding solution $(x(t), y(t))$ of $(2)$ converges to $\left(x_{0}, y_{0}\right)$.

Condition $\left(\mathbf{H}^{*}\right)$ is often automatically satisfied in examples:

Lemma 2.4 Suppose that $c(y)$ and $d(y)$ are affine in $y$. That is, there are two row vectors $c_{0}, c_{1} \in$ $\mathbb{R}^{1 \times n}$ and two scalars $d_{0}, d_{1}$ such that $c(y)=c_{0}+y c_{1}$ and $d(y)=d_{0}+y d_{1}$. Then, Property $(\mathbf{H})$ implies Property $\left(\mathbf{H}^{*}\right)$.

\section{Approximate adaptation}

Perfect adaptation is an ideal mathematical property. In biological systems, regulated behavior may break down due to dilution, turn-over due to gene expression and protein degradation, and other effects, especially over long time intervals. From a modeling viewpoint, it is thus interesting to study mechanisms which provide "approximate" adaptation, in the sense that the response of the system remains approximately constant, as long as parameters (kinetic constants, production rates, degradation rates) stay within appropriate ranges. The reader is referred to the papers [3, 2] for formulations of certain approximate adaptation mechanisms for linear and nonlinear models. This section discusses a few general facts, and works out details for a particular class of systems, to be illustrated with an example in Section 6.5.

One general fact is that a perturbation of the right-hand side of a differential equation results in small perturbations of trajectories, on bounded intervals of time. Specifically, suppose that $x(t)$ is the solution of a set of differential equations $\dot{x}=f(x)$, with initial condition $x(0)=x_{0}$, and consider any fixed time interval $[0, T]$. Next, consider a perturbed equation $\dot{z}=f(z)+h(z)$, and let $z(t)$ be its solution with the same initial condition $z(0)=x_{0}$. Then, if the vector field " $h$ " is small in an appropriate sense (uniformly, for instance, or more generally if its integral along trajectories is small), then it follows that $z(t) \approx x(t)$ for all $t \in[0, T]$; see Theorem 55 in Appendix $\mathrm{C}$ of [23] for details. In principle, and in the absence of additional stability assumptions, the theoretical estimates tend to be conservative, in that the guaranteed approximation is very poor as $T$ increases. However, in practice the approximation may be quite good. As an illustration, consider once again the "sniffer" reactions (3) from [26], and suppose that one perturbs the right-hand side of the equations by adding saturated terms:

$$
\begin{aligned}
\dot{x} & =-k_{4} x+k_{3} u+\frac{V_{1} y}{K_{1}+y} \\
\dot{y} & =-k_{2} x y+k_{1} u+\frac{V_{2} x}{K_{2}+x}
\end{aligned}
$$

representing cross-activating feedbacks. Using the step input from Figure 11(a), Figure 3 compares the results of simulations (starting from the zero initial state) of the original and the perturbed systems, when all constants have been chosen as 1 . Notice that the perturbed system has a response which is quite close to that of the original system, on the given interval. 


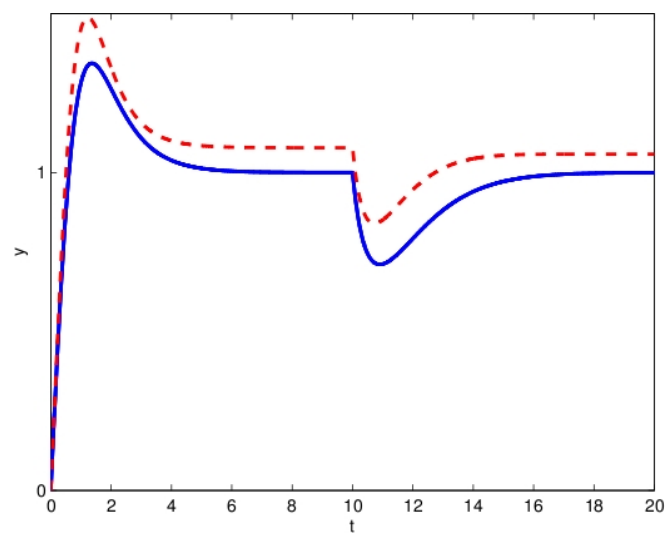

Figure 3: Approximate adaptation: comparison of responses when right-hand side of equation is perturbed as discussed in text. Input is as in Figure 11(a). Solid line is for original system and dashed line for perturbed system.

Another sense of approximate adaptation to step inputs is when adaptation behavior happens only for input signal values in a restricted range. This is illustrated next, using a perturbation of the "sniffer" reactions (3) from [26]. Suppose that the equations are as follows:

$$
\begin{aligned}
& \dot{x}=-k_{4} x+k_{3} u+r(y) \\
& \dot{y}=-k_{2} x y+k_{1} u-p(y) .
\end{aligned}
$$

The term $p(y)$ may represent, for example, a linear or nonlinear degradation effect for the species $y$, while $r(y)$ might represent an activating feedback. (In Section 6.5, it will be shown that a microRNA-based feedforward loop studied in the literature can be represented, after a coordinate change, in this form.) The steady state corresponding to a given constant input $u \equiv u_{0}$ can be found as follows. Setting the right-hand sides of (5) to zero and solving the first equation for $x$ gives $x=\frac{1}{k_{4}}\left(k_{3} u+r(y)\right)$. The expression for $x$ is then substituted in the second equation, to provide the following relation for $u$ in terms of $y$ :

$$
u=Q(y)=\frac{k_{2} y r(y)+k_{4} p(y)}{k_{1} k_{4}-k_{2} k_{3} y}
$$

Suppose that $p(0)=0$ and that $k_{2} y r(y)+k_{4} p(y)$ is an increasing function of $y$ (this happens automatically if, for example, $p(y)$ and $q(y)$ are non-negative and increasing functions of $y$ ). Then $Q$ is an increasing function on the interval $0 \leq y<\alpha=\frac{k_{1} k_{4}}{k_{2} k_{3}}$, with $Q(0)=0$ and a pole at $\alpha$, see Figure 3. The function $Q$ can be then inverted, so as to obtain $y$ as a function of $u, y=Q^{-1}(u)$.

For every step input $u$ whose amplitude is large enough, the steady state value of the response $y$ is close to $\alpha$. In that sense, "approximate" adaptation holds. One way to characterize this effect is as follows. Define $K=Q(\alpha / 2)$. This number plays a role analogous to that of a "half maximal effective concentration" or "EC 50 value" in pharmacology and biochemistry: for any input value $u$ larger than $K, y$ is within $\alpha / 2$ and $\alpha$.

For example, if $p(y)=k y$ (linear degradation/dilution) and $q(y)=0$, then $y=Q^{-1}(u)=\frac{V u}{K+u}$ for appropriate numbers $V$ and $K$. For $u>K$, the response value is within $V / 2$ and $V$. 


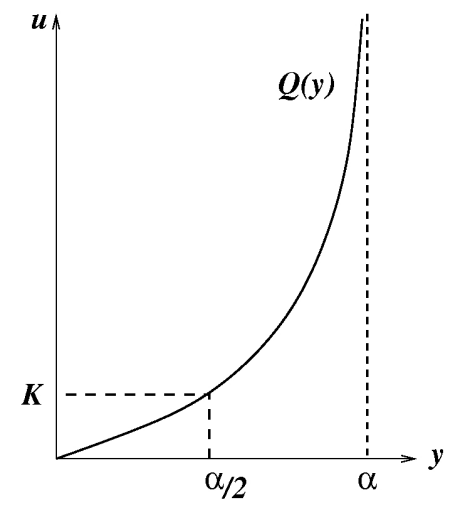

Figure 4: The function $Q$

\section{Pulse memory effects}

As remarked in the Introduction, when a pulse input $u(t)$ is applied to the system (2), the asymptotic value of $y$ does not typically return to its adapted value. There is a "memory" effect as the asymptotic value of $y$ depends on the magnitude of the step. Thus, this section analyzes the effect of a pulse input, that is $u(t)=u_{0} \neq 0$ for some interval $t \in[0, T]$, and $u(t)=0$ for $t>T$. The underlying assumption is that the interval is long enough $(T \gg 1)$ so that $x(T)$ and $y(T)$ are (approximately) in steady state: $x(T) \approx-A^{-1} b u_{0}$ and $y(T) \approx y_{0}$. This means that $x(t) \approx-e^{(t-T) A} A^{-1} b u_{0}$ for $t \geq T$, and $y$ approximately solves $\dot{y}=-c(y) e^{(t-T) A} A^{-1} b u_{0}$ starting at the adapted value $y_{0}$.

Therefore, and changing for simplicity the origin of time to $t=T$, one wishes to estimate the limiting value of the solution of the initial-value problem:

$$
\dot{y}=-c(y) e^{t A} A^{-1} b u_{0}, \quad y(0)=y_{0} .
$$

In general, such a differential equation, even though scalar, is not easy to solve, because of the time dependence. Two special cases are as follows.

\subsection{Affine case}

When $c(y)=c_{0}+y c_{1}$ is affine in $y$, one may write (6) as:

$$
\dot{y}+\alpha(t) y=\beta(t)
$$

where $\alpha(t)=c_{1} e^{t A} A^{-1} b u_{0}$ and $\beta(t)=-c_{0} e^{t A} A^{-1} b u_{0}$. This is a linear differential equation, which can be solved in a standard manner by using the integrating factor $e^{\int \alpha(t) d t}$.

\subsection{Separable case}

Another special case is that in which one may decompose $c(y)$ as follows:

$$
c(y)=\theta(y) c
$$


where $\theta(y)$ is a nowhere vanishing scalar continuous function of $y$, and $c$ is a row vector. Details in this special case are as follows.

Separating variables,

$$
\int_{y_{0}}^{y(t)} \frac{d z}{\theta(z)}=-c\left(\int_{0}^{t} e^{s A} d s\right) A^{-1} b u_{0}=c\left(I-e^{t A}\right) A^{-2} b u_{0} .
$$

The function $\Theta_{0}(y):=\int_{y_{0}}^{y} \frac{d z}{\theta(z)}$ is strictly increasing or strictly decreasing if $\theta$ is positive or negative respectively, so its inverse $\Theta:=\Theta_{0}^{-1}$ is well defined, as it is also strictly increasing or strictly decreasing respectively.

One concludes that:

$$
\hat{y}_{u_{0}}=\Theta\left(c A^{-2} b u_{0}\right)
$$

is the steady-state value of $y$ after the system has been subjected to a long pulse of amplitude $u_{0}$ and the pulse is removed. Recalling the conditions under which $\Theta$ is increasing or decreasing, one may summarize as follows:

Proposition 4.1 Assuming the form in (7), with $\theta(y)$ nonzero and continuous on $\mathcal{Y}$, the steady state value $\hat{y}_{u_{0}}$ is:

(a) an increasing function of $u_{0}$ if $\theta\left(y_{0}\right) c A^{-2} b>0$, and

(b) a decreasing function of $u_{0}$ if $\theta\left(y_{0}\right) c A^{-2} b<0$.

Notice that $\Theta(0)=y_{0}$, so the steady state $\hat{y}_{u_{0}}$ after a pulse is smaller (respectively, larger) than the adaptation steady state $y_{0}$ (that results after a step input) provided that $\theta\left(y_{0}\right) c A^{-2} b<0$ (respectively, $>0$ ).

\subsection{Non-ideal pulses}

Observe that, while the previous analysis concerned only ideal pulses (the value of $u(t)$ returns exactly to zero after time $t=T$ ), approximately the same phenomenon will still occur if $u$ merely returns to a "small" value, in the following sense. Suppose that $u(t)=u_{0} \neq 0$ for some interval $t \in[0, T]$, and $u(t)=\varepsilon$ for $t>T$, where $\varepsilon$ is sufficiently small. The asymptotic value of the response $y$ will eventually converge to the adapted value $y_{0}$, if $\varepsilon \neq 0$. However, if $\varepsilon \approx 0$, this convergence to $y_{0}$ will be extremely slow, as the behavior will be close of that for ideal pulses. Mathematically, this fact is a trivial consequence of the continuous dependence of solutions of differential equations on parameters (on finite time intervals).

To formulate this property precisely, call $z(t)$ the value of $y(t)$ that would correspond to the ideal pulse $(u(t)=0$ for $t>T)$, and $y_{\varepsilon}(t)$ the value that corresponds to the input with $u(t)=\varepsilon$ for $t>T$. The subscript $\varepsilon$ is used to emphasize the dependence on the numerical value of $\varepsilon$. For any fixed time $T^{\prime}>T$, it holds that $y_{\varepsilon}\left(T^{\prime}\right) \approx z\left(T^{\prime}\right)$, in the sense that $y_{\varepsilon}\left(T^{\prime}\right) \rightarrow z\left(T^{\prime}\right)$ as $\varepsilon \rightarrow 0$. (See for example, Theorem 1 in [23] for a proof; explicit estimates of convergence can be obtained using the Gronwall inequality, as discussed in that textbook.)

Another way in which a pulse may differ from an ideal pulse is if the cut-off to $u(t)=0$ is not sharp. In fact, current microfluidics technologies allow one to produce pulsatile-like inputs to 
cell signaling systems, but these might exhibit a slow decay at the tail end. (The same effect may manifest itself in real systems, when an intermediate species stands between the input and the $x$ and $y$ variables.) If the cut-off is sharp enough, a continuity argument similar to the one explained for pulses that sharply return to a constant value $\neq 0$ applies. However, for slower decays to $u=0$, the asymptotic value of the response may indeed return to close to adapted values, as was earlier illustrated with an example, see Figure 1(h).

\section{Proofs}

The following is an elementary observation about scalar differential equations.

Lemma 5.1 Consider the scalar time-dependent differential equation

$$
\dot{y}=F(t, y)+\varphi(y)
$$

where $F$ and $\varphi$ are differentiable functions. Assume that $F(t, y) \rightarrow 0$ as $t \rightarrow \infty$ uniformly on $y \in K$, where $K \subseteq \mathbb{R}$ is a closed and bounded interval, and that there is some $y_{0} \in K$ such that $\varphi(y)>0$ for all $y<y_{0}$ and $\varphi(y)<0$ for all $y>y_{0}$. Then, every solution $y:[0, \infty) \rightarrow K$ is so that $y(t) \rightarrow y_{0}$ as $t \rightarrow \infty$.

Proof. Let $y(t)$ be a solution with values in $K$, and pick any open neighborhood $N$ of $y_{0}$. We must show that, for some $T, y(t) \in N$ for all $t>T$. The set $K \backslash N$ is the union of two closed and bounded sets $A_{-}$and $A_{+}$(either of which might possibly be empty, if $y_{0}$ is an endpoint of $K$ ) such that $y \in A_{-} \Rightarrow y<y_{0}$ and $y \in A_{+} \Rightarrow y>y_{0}$. By continuity of the function $\varphi$, there is some positive number $\delta$ such that $\varphi(y)>\delta$ for all $y \in A_{-}$and $\varphi(y)<-\delta$ for all $y \in A_{+}$. For some $t_{0}$, $|F(t, y)|<\delta / 2$ for all $y \in K \backslash N$ and all $t \geq t_{0}$. Thus, for $t \geq t_{0}$ we have that $\dot{y}(t)>\delta / 2$ if $y(t) \in A_{-}$ and $\dot{y}(t)<-\delta / 2$ if $y(t) \in A_{+}$. This means that for some $T>t_{0}$ it will hold that $y(t)$ exits $K \backslash N$ and does not enter again, as needed.

\section{Proof of Proposition 2.1}

We will apply Lemma 5.1. Suppose that Property $(\mathbf{H})$ holds. Pick any step input $u \equiv u_{0} \neq 0$ and an initial condition of (2), and suppose that the corresponding solution $(x(t), y(t))$ of (2) is so that $y(t)$ is bounded. Since $\mathcal{Y}$ is a closed set, this is the same as saying that $y(t) \in K$ for all $t$, for some closed and bounded interval $K \subseteq \mathcal{Y}$. We have that $x(t)=\theta(t)-A^{-1} b u_{0}$ for all $t \geq 0$, where $\theta(t)=e^{t A}\left(x_{0}+A^{-1} b u_{0}\right)$ and therefore $y$ satisfies:

$$
\dot{y}=F(t, y)+\varphi(y)=c(y) \theta(t)+\varphi(y)
$$

where $\left.\varphi(y)=\left(d(y)-c(y) A^{-1} b\right)\right) u_{0}$. Property $(\mathbf{H})$ gives the property needed for $\varphi$ in the Lemma. On the other hand, $\theta(t) \rightarrow 0$ (as $e^{t A} \rightarrow 0$, by stability), so $F(t, y) \rightarrow 0$ uniformly on $y \in K$. Thus, Lemma 5.1 gives the desired conclusion. 


\section{Proof of Lemma 2.2}

We pick any initial condition, and want to show that the corresponding solution $(x(t), y(t))$ of $(2)$ is such that $y(t)$ is bounded. We will prove that $y(t)$ is upper-bounded, the lower bound proof being similar, and assume that $\mathcal{Y}$ is not upper bounded, since otherwise we are done. By Property $\left(\mathbf{H}^{*}\right)$, we may pick $\bar{\varepsilon}>0$ and $y_{2} \in \mathcal{Y}$ such that $|c(y)| \bar{\varepsilon}+\varphi(y)<0$ for all $y>y_{2}$, where the function $\varphi$ is as in the proof of Proposition 2.1. By (8), we know that $\dot{y}(t)<0$ as long as $y(t)>y_{2}$ and $t \geq t_{0}$, where $t_{0}$ is picked so that $|\theta(t)|<\bar{\varepsilon}$ for all $t \geq t_{0}$. This clearly implies that $y(t)$ is upper bounded.

\section{Proof of Lemma 2.4}

Suppose that $c(y)$ and $d(y)$ are affine in $y, c(y)=c_{0}+y c_{1}$ and $d(y)=d_{0}+y d_{1}$. As Property $(\mathbf{H})$ holds,

$$
\mu+\nu y=d_{0}+y d_{1}-\left(c_{0}+y c_{1}\right) A^{-1} b<0
$$

for $y>y_{0}, y \in \mathcal{Y}$, where we are writing $\mu:=d_{0}-c_{0} A^{-1} b$ and $\nu:=d_{1}-c_{1} A^{-1} b$.

Fix any $u_{0}>0$. We need to show that Property $\left(\mathbf{H}^{*}\right)$ holds. We will show the existence of $\bar{\varepsilon}$ and $y_{2}$; existence of $y_{2}$ is proved in a similar way. Our goal is to pick $y_{2} \in \mathcal{Y}$ in such a way that

$$
|c(y)| \bar{\varepsilon}+u_{0}\left(d(y)-c(y) A^{-1} b\right)<0
$$

whenever $y>y_{2}$ is an element of $\mathcal{Y}$. If the interval $\mathcal{Y}$ is upper bounded, we may pick $y_{2}$ equal to its right endpoint, and this property is satisfied vacuously. Thus, we assume from now on that $\mathcal{Y}$ is not upper bounded.

We claim that $\nu<0$. Indeed, if $\nu \geq 0$ then $\mu+\nu y \geq \mu+\nu y_{0}=0$ for any $y>y_{0}, y \in \mathcal{Y}$, which would contradict Property $(\mathbf{H})$. (Note that there exist such $y>y_{0}$, because $y_{0}$ cannot be the right endpoint of $\mathcal{Y}$, because $\mathcal{Y}$ is not upper bounded.)

For (9) to be satisfied, and assuming we pick $y_{2} \geq 0$, it is enough that this inequality should hold:

$$
\left(\left|c_{0}\right| \bar{\varepsilon}+u_{0} \mu\right)+y\left(\left|c_{1}\right| \bar{\varepsilon}+u_{0} \nu\right)<0
$$

(because $\left.\left|c_{0}+c_{1} y\right| \leq\left|c_{0}\right|+\left|c_{1}\right| y\right)$. We let $\bar{\varepsilon}:=-\frac{\nu}{2\left|c_{1}\right| u_{0}}$. Then, for $y>0$,

$$
\left(\left|c_{0}\right| \bar{\varepsilon}+u_{0} \mu\right)+y\left(\left|c_{1}\right| \bar{\varepsilon}+u_{0} \nu\right)<\left(\left|c_{0}\right| \bar{\varepsilon}+u_{0} \mu\right)+y u_{0} \nu / 2
$$

and, since the upper bound is a linear function with negative slope, it will be negative for large $y$.

\section{Examples}

We show here how the results apply, in particular, to the models in the papers [26] and [30].

\section{1 "Sniffer" model}

The "perfect adaptation" model in 26] is, after a renaming of variables and a slight rearranging to bring into the form $(2)$, as shown in $\sqrt{3}$. Here $y_{0}=\frac{k_{1} k_{4}}{k_{2} k_{3}}$, and $\mathbf{( H )}$ is satisfied because $d(y)-$ 
$c(y) A^{-1} b=k_{1}-\frac{k_{2} k_{3}}{k_{4}} y$ changes sign at $y=y_{0}$. This example has the form in Lemma 2.4, so convergence holds.

To study the effect of pulses, we write $c(y)$ in the form (7) using $c=-k_{2}$ and $\theta(y)=y$. So $\Theta_{0}(y)=\int_{y_{0}}^{y} d z / z=\ln (y)-\ln \left(y_{0}\right)=\ln \left(y / y_{0}\right)$. It follows that $\Theta(r)=y_{0} e^{r}$, so

$$
\hat{y}_{u_{0}}=y_{0} \exp \left(-\frac{k_{2} k_{3}}{k_{4}^{2}} u_{0}\right)
$$

which decreases with $u_{0}$.

\subsection{Dictyostelium chemotaxis models from [30]}

There are several models given in [30], but they all have the same general interpretation. The authors of [30], based on previous work [12], postulate the existence of a "response regulator" R, a variable that correlates to the chemotactic activity of the system, that can be in an "active" or in an "inactive" form. The activation and inactivation of $\mathrm{R}$ are regulated by a pair of opposing processes: an excitation process that induces an increase in the level of the response $R$, and an inhibition process that lowers this response. The input to the system is the extracellular chemoattractant concentration, and it is assumed that this signal triggers increases in concentrations in both the activation and inactivation elements. We denote by $y(t)$ the concentration of active regulator, by $\alpha-y(t)$ the concentration of inactive regulator, where $\alpha$ is the total concentration (active+inactive), assumed constant, and by $x_{1}(t)$ and $x_{2}(t)$ the concentrations of the activation and inactivation elements respectively.

There are several alternative models given in [30]. The first one is, in our notations:

$$
\begin{aligned}
\dot{x}_{1} & =-k_{1} x_{1}+k_{2} u \\
\dot{x}_{2} & =-k_{3} x_{2}+k_{4} u \\
\dot{y} & =k_{5}(\alpha-y) x_{1}-k_{6} y x_{2}
\end{aligned}
$$

where the $k_{i}$ 's are positive constants. This has the form (2), with $\mathcal{X}=\mathbb{R}_{\geq 0}^{2}, \mathcal{Y}=[0, \alpha], c(y)=$ $\left(k_{5}(\alpha-y),-k_{6} y\right)$, and $d(y)=0$. To simplify notations, let us write $P=\frac{k_{2} \bar{k}_{5}}{k_{1}}$ and $Q=\frac{k_{4} k_{6}}{k_{3}}$. Note that Property $(\mathbf{H})$ is satisfied, as the solution $y_{0}$ of

$$
P(\alpha-y)-Q y=0
$$

belongs to the interval $(0, \alpha)$, and the algebraic expression changes there from positive to negative. The $y$-dynamics is bounded, by definition, so we have convergence.

Since the $y$ equation is affine, the memory effect of pulses can be obtained by solving an appropriate linear differential equation, as explained earlier, but the expression for the solution is algebraically very involved. We can, however, make some qualitative remarks.

Lemma 6.1 The solution of the initial-value problem

$$
\dot{y}=P(\alpha-y) e^{-k_{1} t} u_{0}-Q y e^{-k_{3} t} u_{0}, \quad y(0)=y_{0}
$$

has a limit $\hat{y}_{u_{0}}$. If $k_{3}>k_{1}$ then $\hat{y}_{u_{0}}>y_{0}$, and if $k_{3}<k_{1}$ then $\hat{y}_{u_{0}}<y_{0}$. 
Proof. We assume that $k_{3}>k_{1}$; the case $k_{3}<k_{1}$ is proved in an analogous fashion. Since $y(t)$ is bounded, it will be enough to show that there cannot exist two limit points $0 \leq \bar{y}_{1}<\bar{y}_{2} \leq \delta$ of the solution. So assume that such points exist, and let $0<t_{1}<s_{1}<t_{2}<s_{2} \ldots \rightarrow \infty$ be so that $y\left(t_{i}\right) \rightarrow \bar{y}_{1}$ as $i \rightarrow \infty$ and $y\left(s_{i}\right) \rightarrow \bar{y}_{2}$ as $i \rightarrow \infty$. Pick $\varepsilon:=\frac{1}{2}\left(\bar{y}_{2}-\bar{y}_{1}\right)+\left(\alpha-\bar{y}_{2}\right)>0$ and some time $\bar{t}>0$ such that $P \varepsilon>\alpha Q e^{\left(k_{1}-k_{3}\right) \bar{t}}$ (there is such a $\bar{t}$ because $k_{1}-k_{3}<0$ ).

Note the following property:

$$
y(t)<\alpha-\varepsilon \text { and } t \geq \bar{t} \Rightarrow \dot{y}(t)>0 .
$$

Indeed,

$$
\frac{e^{k_{1} t}}{u_{0}} \dot{y}=P(\alpha-y(t))-Q y(t) e^{\left(k_{1}-k_{3}\right) \bar{t}}>P \varepsilon-Q \alpha e^{\left(k_{1}-k_{3}\right) t}>0
$$

because $\alpha-y(t)>\varepsilon$ and $y(t)<\alpha$, so $\dot{y}(t)>0$ as claimed.

Since $\bar{y}_{2}>\alpha-\varepsilon$, there is some $i$ so that $y\left(s_{i}\right)>\alpha-\varepsilon$. Without loss of generality, we may assume that $s_{i}$ was picked larger than $\bar{t}$. Then,

$$
t>s_{i} \Rightarrow y(t) \geq \alpha-\varepsilon \text {. }
$$

(Otherwise, there would exist some $a<\alpha-\delta$ and some $T>s_{i}$ such that $y(T)=a$, and we can assume that $T$ has been picked smallest possible with this property, for the given $a$. Pick $\delta>0$ so that $s_{i}<T-\delta$ and so that the interval $I=(T-\delta, T]$ has the property that $y(I) \subseteq[0, \alpha-\varepsilon)$. Then, by property $(13), \dot{y}(t)>0$ for all $t \in I$. So $y(t)<y(T)$ for all $t \in I$, which contradicts the minimality of $T$.)

On the other hand, since $\bar{y}_{1}<\alpha-\varepsilon, y\left(t_{j}\right)<\alpha-\varepsilon$ for all sufficiently large $j$. This contradicts (14) if $j>i$. The contradiction shows that such $\bar{y}_{1}<\bar{y}_{2}$ cannot exist, so the function $y(t)$ is convergent as $t \rightarrow \infty$.

We must now prove that $\hat{y}_{u_{0}}>y_{0}$. Since $y_{0}$ solves $(11)$, and $e^{\left(k_{1}-k_{3}\right) t}<1$ for all $t \geq 0$, it follows that $P\left(\alpha-y_{0}\right) e^{-k_{1} t}-Q y_{0} e^{-k_{3} t}>0$ for all $t \geq 0$. Thus:

$$
\dot{y}(t)=P(\alpha-y(t)) e^{-k_{1} t}-Q y(t) e^{-k_{3} t} \geq P\left(\alpha-y_{0}\right) e^{-k_{1} t}-Q y_{0} e^{-k_{3} t}>0
$$

whenever $t \geq 0$ is such that $y(t) \leq y_{0}$. This implies that $y(t)>y(0)=y_{0}$ for all $t>0$. Therefore the limit also satisfies $\hat{y}_{u_{0}}>y_{0}$.

The interpretation of Lemma 6.1 is obvious: $k_{3}>k_{1}$ means that the inhibitor $\left(x_{2}\right)$ degrades at a faster rate than the activator $\left(x_{1}\right)$. Thus, when the external signal is turned-off, there is a residual effect due to the additional activator still present, which implies a positive memory effect, in the sense that the response is higher than its value at $u=0$. Similarly, when $k_{3}<k_{1}$, there is additional repressor present and the memory effect is negative (that is, lower than when $u=0$ ).

The other models from [30] are similar, differing only in the placement of the feedforward terms in the $y$ equation, and the stability results apply equally well. Let us consider one of the variants:

$$
\begin{aligned}
\dot{x}_{1} & =-k_{1} x_{1}+k_{2} u \\
\dot{x}_{2} & =-k_{3} x_{2}+k_{4} x_{1} \\
\dot{y} & =k_{5}(\alpha-y) u-k_{6} y x_{2} .
\end{aligned}
$$

Note that the activator now acts so as to enhance the inhibitor, and the input signal acts directly on the response element. This has the form $(2)$, with $\mathcal{X}=\mathbb{R}_{\geq 0}^{2}, \mathcal{Y}=[0, \alpha], c(y)=\left(0,-k_{6} y\right)$, and 
$d(y)=k_{5}(\alpha-y)$. Note that Property $(\mathbf{H})$ is satisfied, as $k_{5}(\alpha-y)-\frac{k_{2} k_{4} k_{6}}{k_{1} k_{3}} y$ changes sign at a $y_{0} \in(0, \alpha)$. The $y$-dynamics is bounded, by definition, so we have convergence.

Once again, since the $y$ equation is affine, the memory effect of pulses can be computed using linear differential equations theory. The memory effect is in this case decreasing in $u_{0}$, independently of the parameters $k_{i}>0$. This is because the initial value problem after the signal has been turnedoff has the form:

$$
\dot{y}(t)=-y(t) \theta(t)
$$

for some positive function $\theta(t)$, so $y(t)<0$ for all $t$. Intuitively: the activation effect $(u)$ turns-off immediately, but there is a residual inhibition effect $\left(x_{2}\right)$.

As an concrete illustration, let us work out the case in which all constants are equal to 1 . In this case, solving $(1-y)-y=0$ gives $y_{0}=1 / 2$, and $\tilde{y}_{u_{0}}$ is the limiting value of the solution of:

$$
\dot{y}=-y e^{-t}(t+1) u_{0}
$$

(because $\left.x_{2}(t)=e^{-t}(t+1) u_{0}\right)$. Thus, $y(t)=\frac{1}{2} e^{\left[(2+t) e^{-t}-2\right] u_{0}}$ and therefore $\hat{y}_{u_{0}}=\frac{1}{2} e^{-2 u_{0}}$, so that, indeed, the memory effect is negative.

\subsection{A Michaelis-Menten model}

Non-affine variant of the above examples may be obtained by using Michaelis-Menten dynamics for activation and inhibition reactions. For instance, we may write:

$$
\dot{y}=\frac{V_{1}(\alpha-y)}{K_{1}+(\alpha-y)} u-\frac{V_{2} y}{K_{2}+y} x_{2}
$$

for some positive constants $V_{i}$ and $K_{i}$. Once again, our hypotheses apply, and there is convergence to $y_{0}$.

We study memory effects for pulses for this example, but only in the special case in which all constants are equal to 1 and for a 1 -dimensional $x$-system:

$$
\begin{aligned}
\dot{x} & =-x+u \\
\dot{y} & =\frac{(1-y)}{1+(1-y)} u-\frac{y}{1+y} x .
\end{aligned}
$$

We have that $y_{0}=1 / 2$ and $x_{0}=u_{0}$, and $c(y)=-y /(1+y)$. We are in the separable situation described earlier, and so solve $\dot{y}=c(y) e^{-t} u_{0}$ using separation of variables. Writing

$$
\ln y(t)+\ln 2+y(t)-1 / 2=\int_{1 / 2}^{y(t)}\left(\frac{1}{s}+1\right) d s=-\int_{0}^{t} e^{-s} d s u_{0}=\left(e^{-t}-1\right) u_{0}
$$

we have, taking limits, that $\hat{y}_{u_{0}}$ is the solution of the algebraic equation:

$$
\ln \hat{y}_{u_{0}}+\hat{y}_{u_{0}}=1 / 2-\ln 2-u_{0}
$$

and so decreases with $u_{0}$. For example, if $u_{0}=1$ then $\hat{y}_{u_{0}} \approx 0.24$, which is less than one-half of the adapted value $y_{0}=0.5$. 


\subsection{A carbohydrate uptake system}

The paper [1] deals with a feedforward motif that appears in one of the carbohydrate uptake systems in E.coli, the phosphotransferase system (PTS) for glucose uptake involving phosphoenolpyruvate (PEP). The reactions in this system result in the phosphorylation of Enzyme II A. "EIIA-P" is used to denote the phosphorylated form of this enzyme, which in turn has various regulatory functions through synthesis of cAMP. A feedforward loop is obtained when viewing $u=$ Glc6P (glucose 6 -phosphate) as input to the system, and using as state variables the concentrations $x=\mathrm{TP}$ (triose phosphate) and $y=$ PEP. See Figure 2 in that paper: there are positive effects of $u$ on $x$, and of $x$ on $y$, as well as a countering negative direct effect of $u$ on $y$ that involves the dephosphorylation of PEP into Prv via pyruvate kinase. For a simplified analysis following [11], assuming equation (1) from that paper, there results that the output of the system, the concentration of EIIA-P, is an increasing function of the ratio of concentrations PEP/Prv, where Prv is pyruvate (equations (3) and (5) in the citation). The objective of keeping EIIA-P approximately constant is achieved if the ratio PEP/Prv is kept approximately constant. The model in [11, Supplemental Materials, equations $(2,3,11)$ together with the assumption that equation (1) in the paper holds, provides the following equations for $x$ and $y$ :

$$
\begin{aligned}
& \dot{x}=-a x+b u+\alpha \\
& \dot{y}=a x-p u y-\beta
\end{aligned}
$$

assuming that the simplest mass-action model is used for supplemental equation (12) of the reference. The constants $\alpha$ and $\beta$ represent uptake rates (supplementary equations $(8,9)$ ). In the case when $\alpha=\beta=0$, there results a system of the general form (2), with $A=a, c(y)=a$ and $d(y)=-p y$ (affine case). Solutions for constant nonzero inputs have $y(t) \rightarrow y_{0}=b / p$, and the effect of pulses can be analyzed easily. When $\alpha$ and $\beta$ are nonzero, the adaptation property fails, although it holds approximately if these numbers are small. However, even for large $\alpha, \beta$, the steady state when $u$ is a constant equal to $u_{0}$ is:

$$
y_{0}=\frac{b}{p}+\frac{\alpha-\beta}{p u_{0}} .
$$

This is still approximately constant, $y_{0} \approx b / p$, provided that $u_{0}$ be sufficiently large, just as in the previous discussion of approximate adaptation.

\subsection{An example of approximate adaptation}

The paper [29] provides the following model of microRNA-mediated feedforward adaptation:

$$
\begin{aligned}
\dot{p}_{1} & =\alpha_{1} \omega-\beta_{1} p_{1} \\
\dot{m}_{1} & =\frac{\alpha_{2} p_{1}^{m}}{1+p_{1}^{m}}-\gamma m_{1} m_{2}-\beta_{2} m_{1} \\
\dot{m}_{2} & =\frac{\alpha_{3} p_{1}^{m}}{1+p_{1}^{m}}-\gamma m_{1} m_{2}-\beta_{3} m_{2} \\
\dot{p}_{2} & =\alpha_{4} m_{2}-\beta_{4} p_{2}
\end{aligned}
$$

where $p_{1}, m_{1}, m_{2}, p_{2}, \omega$ are respectively the species concentrations of an "upstream factor", a microRNA, a target mRNA, the protein produced by the target mRNA, and an inducer of the upstream 
factor. The various constants represent transcription, translation, and degradation rates as well as well as the efficiency of pairing of the microRNA to its target. (As in the reference, we pick identical Hill coefficients for both promoters.) The interest in [29] is in studying the robustness of the steady state value of $p_{2}$. Since this value is directly proportional to the steady state value of $m_{2}$, we omit

$p_{2}$ from the model from now on. Similarly, as $\frac{p_{1}^{m}}{1+p_{1}^{m}}$ is an increasing function of $p_{1}$, which is in turn proportional to $\omega$ at steady state, we will think of this term as an input " $u$ " and drop the equation for $p_{1}$ as well. We are left with the following two-dimensional $\left(m_{1}, m_{2}\right)$ system:

$$
\begin{aligned}
& \dot{m}_{1}=\alpha_{2} u-\gamma m_{1} m_{2}-\beta_{2} m_{1} \\
& \dot{m}_{2}=\alpha_{3} u-\gamma m_{1} m_{2}-\beta_{3} m_{2} .
\end{aligned}
$$

When expressed in the alternative coordinates $x=m_{1}-m_{2}$ and $y=m_{2}$, the system has the form in Equations (5), with $k_{1}=\alpha_{3}, k_{2}=\gamma, k_{3}=\alpha_{2}-\alpha_{3}, k_{4}=\beta_{2}, r(y)=\left(\beta_{3}-\beta_{2}\right) y$, and $p(y)=\gamma y^{2}+\beta_{3} y$. Thus this system exhibits an approximately adaptive behavior for large inputs, as discussed in Section 3. In particular, consider the parameter values used in [29]: $\alpha_{1}=0.01$, $\alpha_{2}=0.1, \alpha_{3}=0.02, \alpha_{4}=0.01, \beta_{1}=0.001, \beta_{2}=0.0025, \beta_{3}=0.002, \beta_{4}=0.001$, and $\gamma_{=0} 001$. Then $Q(y)=\frac{0.000002 y^{2}+0.000005 y}{0.00005-0.00008 y}$ and, with the notations in Section 3 , the "adaptation" value for $m_{2}$ is $\alpha=0.625$ with the " $\mathrm{EC}_{50}$ value" $K=Q(\alpha / 2) \approx 0.07$. The corresponding steady state value of $p_{2}$ is $\alpha_{4} m_{2} / \beta_{4}=6.25$. (Compare Figure 2 in [29].)

\section{Remarks on integral feedback}

The "internal model principle" (see e.g. [24]) states that, if a system perfectly adapts to all step inputs, then it may be re-written, possibly after performing a nonlinear change of coordinates, as a system in which an integral of the regulated quantity (response variable) is fed-back. A feedforward (not feedback) system that exhibits perfect adaptation might appear at first sight to be a counterexample to this fact. However, there is not necessarily a contradiction, as a change of coordinates may allow one to transform a feedforward into a feedback system. This observation was made in [9], and we discuss it further here through an example.

As an illustration, consider the following two-dimensional linear system:

$$
\begin{aligned}
\dot{x} & =-x+u \\
\dot{y} & =-x-a y+u
\end{aligned}
$$

(where " $a$ " is some positive constant). This system has the property, when the input $u$ is constantly equal to a value $u_{0}$, that every solution converges to the state $\left(u_{0}, 0\right)$ as $t \rightarrow \infty$. Thus, the response variable $y(t)$ converges to $y_{0}=0$ no matter what is the actual value of $u_{0}$. The system response is perfectly adaptative.

The system (16) has a feedforward form. However, the same system can be recast as an integral feedback system, as follows. Suppose that we choose to represent the system using the state variables $z=x-y$ and $y$ instead of $x$ and $y$. In the new set of coordinates:

$$
\begin{aligned}
& \dot{z}=a y \\
& \dot{y}=-z-(a+1) y+u
\end{aligned}
$$

which can be viewed as a system in which the rate of change of the regulated quantity $y$ depends on $y$ itself (proportional negative feedback) as well as on $z$, which is (up to a positive constant multiple) the integral of $y$ (integral feedback term). 
Notice that, especially when seen as an integral feedback system, it is immediately obvious that for every step input $u \equiv u_{0}$ (not merely nonzero steps), any steady state has the value $y=0$, since $0=\dot{z}=a y$ at steady states. So, after a pulse, the system will eventually also converge to the adapted value (since we can see the behavior, after the end of the pulse, as the behavior corresponding to a zero step). Thus, the memory effect discussed in this note will not occur for a true integral feedback system such as the linear system shown above. (Note that the system (16) does not have the exact form (2) studied in this paper, because of the additive, not multiplicative, term "-ay".)

As an example, the plot shown in Figure 7(b) shows the response to the pulse in Figure 7(a), for system (16) with $a=0.1$. Adaptation to $y=0$ results in this case, which also happens with the response in Figure 7(d) to the step input in Figure 7(c). (Scales for $y$ have been normalized, so as to show relative changes. The response in (d) eventually settles back to zero, not shown.) Note that the adaptation to the pulse is faster than that for the step input. Interestingly, this

(a)

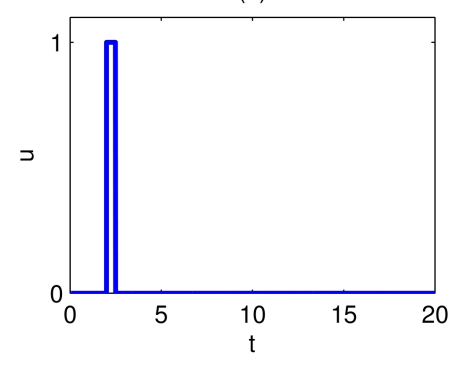

(c)

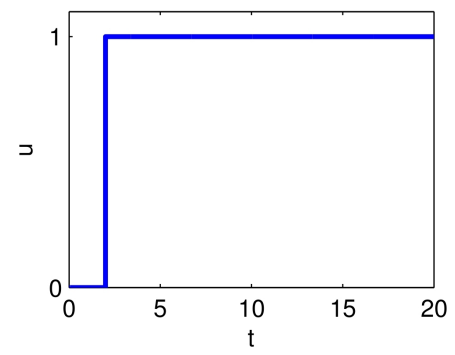

(b)

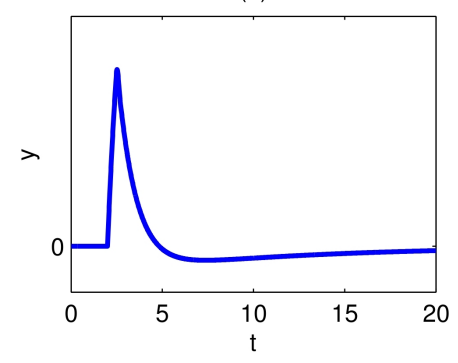

(d)

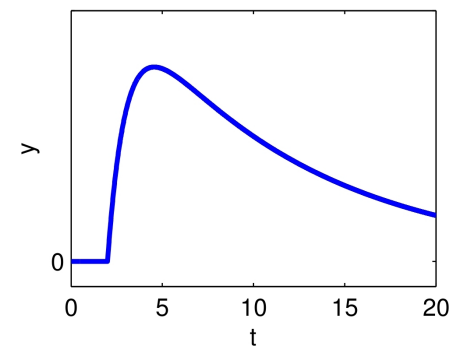

Figure 5: Responses to a pulse and to a step input, for a linear feedforward system discussed in the text. (a) Pulse input. (b) Response to the input in (a). (c) Step input. (d) Response to the input in $(\mathrm{c})$.

model reproduces qualitatively Figure 2 from [4] reproduced as Figure 7 in this paper. The figure compares the changes in translocation of CRAC (cytosolic regulator of adenylyl cyclase), reported by relative fluorescence of a CRAC-GFP construct, in chemotactic Dictyostelium in response to a "short" (i.e., pulse) or a "continuous" (i.e., step) stimulus generated of cAMP.

The integral feedback form (17) is often said to be more "robust" for adaptation than the feedforward form (16), because the steady-state response $y$ is still zero even if the second equation is arbitrary modified: if $\dot{z}=0$ and $a \neq 0$, one has that $y=0$. In contrast, modifications in (16) affect the steady state value of $y$. This claim of robustness is very misleading, however, because perturbations of the first equation in (17) will generally change the steady state value of $y$.

The connection to the theory in [24] is somewhat subtle. It is shown there that, under appropriate

\footnotetext{
*The author thanks Pablo Iglesias for pointing out this reference.
} 


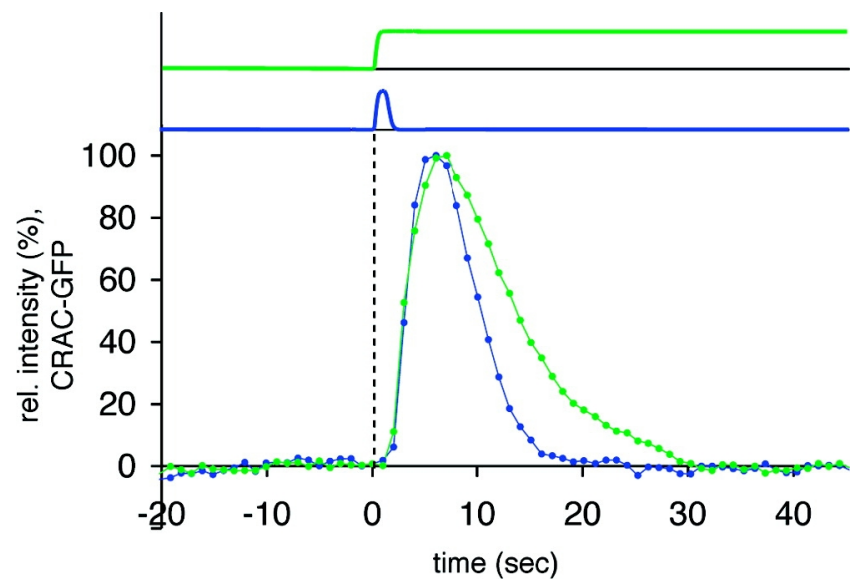

Figure 6: Reproduced (microscopy inset removed) with permission from Figure 2 in [4]. Responses to step and pulse cAMP input in Dictyostelium, as discussed in text. The response to the pulse input settles faster than the response to the step.

technical restrictions on the dynamics, even a nonlinear system that adapts to all step inputs can be recast as an integral feedback system. The key is the assumption "all inputs" - the recasting may fail to be global when inputs are restricted. Rather than explaining here the nonlinear theory, a simple local (linearized) version is analyzed next as an illustration of these ideas.

Once again, take as an illustration the "sniffer" equations from [26] given in (3). For simplicity of notations (nothing much changes in the general case), take all kinetic constants equal to one:

$$
\begin{aligned}
& \dot{x}=-x+u \\
& \dot{y}=-y x+u .
\end{aligned}
$$

Suppose that one is only interested in studying the behavior of this system in the vicinity of the steady state $(\bar{x}, \bar{y})=(a, 1)$ and the step input $u(t) \equiv a$ for all $t$. For small changes in initial states and input values, the system is well-approximated by its linearization around these values, that is, the system that is obtained when replacing the nonlinear term " $y x$ " by

$$
(\partial(y x) / \partial x) x+(\partial(y x) / \partial y) y
$$

where the partial derivatives are understood as evaluated at $(\bar{x}, \bar{y})$. Since $\partial(y x) / \partial x=\bar{y}=1$ and $\partial(y x) / \partial y=\bar{x}=a$, we have that the linearized system is precisely the system (16). Thus, so long as $a \neq 0$, the system can be recast (locally) as an integral feedback system. However, in the special case when $a=0$, the recast system has the form $\dot{z}=0, \dot{y}=-z-y+u$. This system is not an integral feedback system, since $z$ no longer contains information about the integral of $y$. (Mathematically, there is now a zero eigenvalue; thus, the system is no longer asymptotically stable, and hence no regulation property holds.)

\section{Discussion}

Adaptation is a feature often exhibited by biological systems, as discussed in the cited references. This paper started from the well-known observation that certain types of feedforward circuits proposed in biological models have adaptation properties, and established rigorous mathematical results along those lines. 
Perhaps of more interest, it was shown that a "memory" effect is often displayed after pulses. The magnitude of this effect is a nonlinear function of the magnitude of the pulse, and estimates were given of its value.

One may speculate regarding what beneficial roles these memory effects of pulses might play. In at least some of the examples, the calculations show that after a very high-amplitude pulse is turned-off, the response settles down to a close to "relaxed" steady state. In the context of a complex system, this response might be appropriate, for example, in a situation in which resources, used up while responding to a large external input, need to be replenished, and this is achieved by turning-off processes controlled by the feedforward circuit; in this way, a "refractory period" would be established.

Of course, the "memory" effect of pulses may or may not play a role in real systems, because parameter ranges may be such that the effect is negligible, or because sharp cutoffs of signals are rare in nature. It remains to see if feedforward circuits function in these regimes, in any real systems.

From an experimental viewpoint, the results in this paper suggest that one might be able to use the pulse-memory property as a way to experimentally distinguish true integral feedback systems from feedforward ones, through the testing of system responses against ideal pulses.

\section{Acknowledgments}

The author wishes to thank Yuan Wang and Pablo Iglesias, as well as two anonymous reviewers, for many useful comments on the manuscript. This research was supported in part by grants from AFOSR, NSF, and NIH, and was carried out in part while visiting the Laboratory for Information and Decision Systems at MIT.

\section{References}

[1] U. Alon. An Introduction to Systems Biology: Design Principles of Biological Circuits. Chapman \& Hall, 2006.

[2] B. Andrews, P. Iglesias, and E.D. Sontag. Signal detection and approximate adaptation implies an approximate internal model. In Proc. IEEE Conf. Decision and Control, San Diego, Dec. 2006, pages 2364-2369. IEEE, 2006.

[3] B. Andrews, E.D. Sontag, and P. Iglesias. An approximate internal model principle: Applications to nonlinear models of biological systems. In Proc. 17th IFAC World Congress, Seoul, pages Paper FrB25.3, 6 pages, 2008.

[4] Carsten Beta, Danica Wyatt, Wouter-Jan Rappel, and Eberhard Bodenschatz. Flow photolysis for spatiotemporal stimulation of single cells. Analytical Chemistry, 79(10):3940-3944, 2007.

[5] A. Cournac and J. A. Sepulchre. Simple molecular networks that respond optimally to timeperiodic stimulation. BMC Syst Biol, 3:29, 2009.

[6] B.A. Francis and W.M. Wonham. The internal model principle for linear multivariable regulators. Appl. Math. Optim., 2:170-194, 1975. 
[7] J. S. A. Hepburn and W. M. Wonham. Error feedback and internal models on differentiable manifolds. IEEE Trans. Automatic Control, 29:397-403, 1984.

[8] G. Hornung and N. Barkai. Noise propagation and signaling sensitivity in biological networks: a role for positive feedback. PLoS Comput. Biol., 4:e8, Jan 2008.

[9] P.A. Iglesias. Feedback control in intracellular signaling pathways: Regulating chemotaxis in dictyostelium discoideum. European J. Control., 9:216225, 2003.

[10] D. Kim, Y. K. Kwon, and K. H. Cho. The biphasic behavior of incoherent feed-forward loops in biomolecular regulatory networks. Bioessays, 30:1204-1211, Nov 2008.

[11] A. Kremling, K. Bettenbrock, and E. D. Gilles. A feed-forward loop guarantees robust behavior in escherichia coli carbohydrate uptake. Bioinformatics, 24:704-710, 2008.

[12] A. Levchenko and P.A. Iglesias. Models of eukaryotic gradient sensing: Application to chemotaxis of amoebae and neutrophils. Biophys J., 82:50-63, 2002.

[13] A. Ma'ayan, S. L. Jenkins, S. Neves, A. Hasseldine, E. Grace, B. Dubin-Thaler, N. J. Eungdamrong, G. Weng, P. T. Ram, J. J. Rice, A. Kershenbaum, G. A. Stolovitzky, R. D. Blitzer, and R. Iyengar. Formation of regulatory patterns during signal propagation in a Mammalian cellular network. Science, 309:1078-1083, Aug 2005.

[14] M. P. Mahaut-Smith, S. J. Ennion, M. G. Rolf, and R. J. Evans. ADP is not an agonist at P2X(1) receptors: evidence for separate receptors stimulated by ATP and ADP on human platelets. Br. J. Pharmacol., 131:108-114, Sep 2000.

[15] S. Mangan, S. Itzkovitz, A. Zaslaver, and U. Alon. The incoherent feed-forward loop accelerates the response-time of the gal system of Escherichia coli. J. Mol. Biol., 356:1073-1081, Mar 2006.

[16] S. Marsigliante, M. G. Elia, B. Di Jeso, S. Greco, A. Muscella, and C. Storelli. Increase of $[\mathrm{Ca}(2+)](\mathrm{i})$ via activation of ATP receptors in PC-Cl3 rat thyroid cell line. Cell. Signal., 14:61-67, Jan 2002.

[17] P. Menè, G. Pugliese, F. Pricci, U. Di Mario, G. A. Cinotti, and F. Pugliese. High glucose level inhibits capacitative Ca2+ influx in cultured rat mesangial cells by a protein kinase Cdependent mechanism. Diabetologia, 40:521-527, May 1997.

[18] T. Nagashima, H. Shimodaira, K. Ide, T. Nakakuki, Y. Tani, K. Takahashi, N. Yumoto, and M. Hatakeyama. Quantitative transcriptional control of ErbB receptor signaling undergoes graded to biphasic response for cell differentiation. J. Biol. Chem., 282:4045-4056, Feb 2007.

[19] R. Nesher and E. Cerasi. Modeling phasic insulin release: immediate and time-dependent effects of glucose. Diabetes, 51 Suppl 1:S53-59, Feb 2002.

[20] L. A. Ridnour, A. N. Windhausen, J. S. Isenberg, N. Yeung, D. D. Thomas, M. P. Vitek, D. D. Roberts, and D. A. Wink. Nitric oxide regulates matrix metalloproteinase-9 activity by guanylyl-cyclase-dependent and -independent pathways. Proc. Natl. Acad. Sci. U.S.A., 104:16898-16903, Oct 2007.

[21] S. Sasagawa, Y. Ozaki, K. Fujita, and S. Kuroda. Prediction and validation of the distinct dynamics of transient and sustained ERK activation. Nat. Cell Biol., 7:365-373, Apr 2005. 
[22] S. Semsey, S. Krishna, K. Sneppen, and S. Adhya. Signal integration in the galactose network of Escherichia coli. Mol. Microbiol., 65:465-476, Jul 2007.

[23] E.D. Sontag. Mathematical Control Theory. Deterministic Finite-Dimensional Systems, volume 6 of Texts in Applied Mathematics. Springer-Verlag, New York, second edition, 1998.

[24] E.D. Sontag. Adaptation and regulation with signal detection implies internal model. Systems Control Lett., 50(2):119-126, 2003.

[25] J. Tsang, J. Zhu, and A. van Oudenaarden. MicroRNA-mediated feedback and feedforward loops are recurrent network motifs in mammals. Mol. Cell, 26:753-767, Jun 2007.

[26] J.J. Tyson, K. Chen, and B. Novak. Sniffers, buzzers, toggles, and blinkers: dynamics of regulatory and signaling pathways in the cell. Curr. Opin. Cell. Biol., 15:221-231, 2003.

[27] E. Voit, A. R. Neves, and H. Santos. The intricate side of systems biology. Proc. Natl. Acad. Sci. U.S.A., 103:9452-9457, Jun 2006.

[28] M. E. Wall, M. J. Dunlop, and W. S. Hlavacek. Multiple functions of a feed-forward-loop gene circuit. J. Mol. Biol., 349:501-514, Jun 2005.

[29] F.-D. Xu, Z.-R. Liu, Z.-Y. Zhang, and J.-W. Shen. Robust and adaptive microRNA-mediated incoherent feedforward motifs. Chinese Physics Letters, 26(2):028701-3, February 2009.

[30] L. Yang and P.A. Iglesias. Positive feedback may cause the biphasic response observed in the chemoattractant-induced response of dictyostelium cells. Systems Control Lett., 55(4):329-337, 2006.

[31] T.-M. Yi, Y. Huang, M.I. Simon, and J. Doyle. Robust perfect adaptation in bacterial chemotaxis through integral feedback control. Proc. Natl. Acad. Sci. USA, 97:4649-4653, 2000. 